\title{
Improvement of survival in Polish stroke patients is related to reduced stroke severity and better control of risk factors: the Krakow Stroke Database
}

\author{
Marta Swarowska, Jacek Burkot, Aleksandra Janowska, Aleksandra Klimkowicz-Mrowiec, \\ Joanna Pera, Agnieszka Slowik, Tomasz Dziedzic
}

Department of Neurology, Jagiellonian University Medical College, Krakow, Poland

Submitted: 26 August 2014

Accepted: 27 September 2014

Arch Med Sci 2016; 12, 3: 552-555

DOI: 10.5114 /aoms.2016.59928

Copyright (C) 2016 Termedia \& Banach

\section{Abstract}

Introduction: In the last decade, the stroke mortality rate in Poland significantly decreased. We hypothesised that stroke severity, the major determinant of outcome, is lowered in Polish stroke patients.

Material and methods: We compared the stroke severity in two cohorts of first-ever ischaemic stroke patients admitted within $24 \mathrm{~h}$ after stroke onset to the Department of Neurology, Jagiellonian University, Krakow in the years 1994-2000 and 2008-2012. To assess stroke severity we used the National Institute of Health Stroke Scale (NIHSS). We defined mild stroke as an NIHSS score $\leq 4$.

Results: We included 816 patients hospitalised in the years 1994-2000 and 569 patients hospitalised in the years 2008-2012. NIHSS score on admission was higher in the former (mean: $12.0 \pm 7.0$ vs. $8.0 \pm 6.0, p<0.01$ ), and the frequency of mild stroke was higher in the latter $(12.7 \%$ vs. $41.8 \%, p<$ $0.01)$. Although the frequency of hypertension ( $67.3 \%$ vs. $81.2 \%, p<0.01)$, diabetes mellitus $(20.8 \%$ vs. $26.4 \%, p=0.02)$ and atrial fibrillation $(20.7 \%$ vs. $26.2 \%, p=0.02$ ) was higher in patients hospitalised in the years $2008-$ 2012 , the systolic and diastolic blood pressure values and the frequency of fasting hyperglycaemia were lower in this cohort. This cohort also less frequently suffered from hypercholesterolaemia $(25.4 \%$ vs. $16.3 \%, p<0.01)$. Conclusions: Reduced stroke severity is associated with better recognition and control of risk factors and explains the improvement of survival in Polish stroke patients.

Key words: ischaemic stroke, stroke severity, risk factors, hypertension, hypercholesterolaemia.

\section{Introduction}

Epidemiological and hospital-based studies conducted in the 1990s showed a higher mortality rate in Polish stroke patients compared to patients from the USA or western European countries [1-3]. In the Warsaw Stroke Registry, 30-day and 1-year case fatality rates in the years 1991-1992 were $43 \%$ and $59.7 \%$, respectively. This excessive stroke mortality was attributed, at least partially, to more severe stroke and higher frequency of cardiovascular diseases (hypertension, atrial fibrillation, diabetes, coronary heart disease, heart failure) in the Polish population [4].

\author{
Corresponding author: \\ Tomasz Dziedzic MD \\ Department of Neurology \\ Jagiellonian University \\ Medical College \\ 3 Botaniczna St \\ 31-503 Krakow, Poland \\ Phone: +48124248600 \\ E-mail: dziedzic@ \\ cm-uj.krakow.pl
}


In the last two decades the death rate from coronary heart disease in Poland halved [5-8]. This reduction was attributed to favourable changes in cardiovascular risk factors ( $54 \%$ fall) and modern treatments ( $37 \%$ fall) [8]. In the last decade a significant decrease in mortality rates was observed in Polish stroke patients $[9,10]$. According to the European Register of Stroke Project, 30-day case fatality in the Warsaw population in the year 2005 was $14.9 \%$ and 1 -year case fatality was $33.1 \%$.

We hypothesised that reduced stroke mortality in Polish patients could be related to decreased stroke severity, which is the major determinant of outcome in stroke patients.

\section{Material and methods}

Using our stroke database, we compared the baseline characteristics of the cohort of patients hospitalised in the Department of Neurology, JagielIonian University, Krakow in the years 1994-2000 and the cohort of patients hospitalised in the years 2008-2012. Consecutive patients with first-ever ischaemic stroke admitted to our stroke unit within $24 \mathrm{~h}$ after stroke onset were eligible for the study. The cohort of patients hospitalised in the years 1994-2000 included 816 persons and the cohort of patients hospitalised in the years 2008-2012 included 569 persons.

All patients underwent head computed tomography (CT) scan within $24 \mathrm{~h}$ after stroke onset. We assessed stroke severity using the Scandinavian Stroke Scale (SSS) [11] for patients hospitalised in the years 1994-2000 and the National Institute of Health Stroke Scale (NIHSS) [12] for patients hospitalised in the years 2008-2012. Nowadays
NIHSS is routinely used in clinical practice; however, for long time this scale was used mainly in the United States, whereas European countries tended to use the SSS. To facilitate the comparisons between groups, we converted SSS scores to NIHSS scores using a published mathematical equation [13]. A previous study demonstrated that total scores for SSS and NIHSS may be interconverted with good precision [13]. We defined mild stroke as an NIHSS score $\leq 4$. This threshold has been commonly used in previous studies on thrombolysis in stroke patients [14].

Arterial hypertension was diagnosed when its presence was documented in medical records or when at least 2 readings of blood pressure were $\geq 140 \mathrm{~mm} \mathrm{Hg}$ (systolic) or $\geq 90 \mathrm{~mm} \mathrm{Hg}$ (diastolic) after the acute phase of stroke. Diabetes mellitus was diagnosed if its presence was documented in medical records or the patient was taking insulin or oral hypoglycaemic agents. Hypercholesterolaemia was defined as a fasting total cholesterol level $>6.1 \mathrm{mmol} / \mathrm{l}$. Fasting hyperglycaemia was defined as a serum glucose level $>6.1 \mathrm{mmol} / \mathrm{l}$. The glomerular filtration rate (GFR) was estimated from the Modification of Diet in Renal Disease equation.

The study protocol was approved by the Bioethics Committee of Jagiellonian University (120/B/2012).

\section{Statistical analysis}

The $\chi^{2}$ test was used to compare proportions and the Mann-Whitney test to compare continuous variables between groups. The calculations were performed using the program Statistica for Windows (version 10, StatSoft, Poland).

Table I. Characteristics of two stroke patient cohorts

\begin{tabular}{|c|c|c|c|}
\hline Parameter & $\begin{array}{l}\text { Cohort } 1994-2000 \\
\qquad(N=816)\end{array}$ & $\begin{array}{l}\text { Cohort } 2008-2012 \\
\qquad(N=569)\end{array}$ & $P$-value \\
\hline Age, mean $\pm S D$ & $69.6 \pm 12.6$ & $70.4 \pm 13.4$ & 0.23 \\
\hline Female, $n(\%)$ & $431(52.8)$ & $296(52.0)$ & 0.77 \\
\hline Hypertension, $n$ (\%) & $549(67.3)$ & $462(81.2)$ & $<0.01$ \\
\hline Diabetes mellitus, $n$ (\%) & $170(20.8)$ & $150(26.4)$ & 0.02 \\
\hline Previous myocardial infarction, $n(\%)$ & $104(12.7)$ & $80(14.1)$ & 0.48 \\
\hline Atrial fibrillation, $n(\%)$ & $169(20.7)$ & $149(26.2)$ & 0.02 \\
\hline Hypercholesterolaemia, $n$ (\%) & $207(25.4)$ & $93(16.3)$ & $<0.01$ \\
\hline TIA prior to stroke, $n(\%)$ & $68(8.3)$ & $30(5.3)$ & 0.03 \\
\hline NIHSS score on admission, mean \pm SD & $12.0 \pm 7.0$ & $8.0 \pm 6.0$ & $<0.01$ \\
\hline Mild stroke, $n(\%)$ & $104(12.7)$ & $238(41.8)$ & $<0.01$ \\
\hline 30-day case fatality, $n(\%)$ & $134(16.4)$ & $30(5.3)$ & $<0.01$ \\
\hline Systolic blood pressure on admission, mean \pm SD & $161 \pm 29$ & $151 \pm 26$ & $<0.01$ \\
\hline Diastolic blood pressure on admission, mean \pm SD & $94 \pm 16$ & $87 \pm 14$ & $<0.01$ \\
\hline Fasting hyperglycaemia, $n$ (\%) & $333(40.8)$ & $180(31.6)$ & $<0.01$ \\
\hline GFR $<60 \mathrm{ml} / \mathrm{min}$ per $1.73 \mathrm{~m}^{2}, n(\%)$ & $300(36.7)$ & $115(20.2)$ & $<0.01$ \\
\hline
\end{tabular}




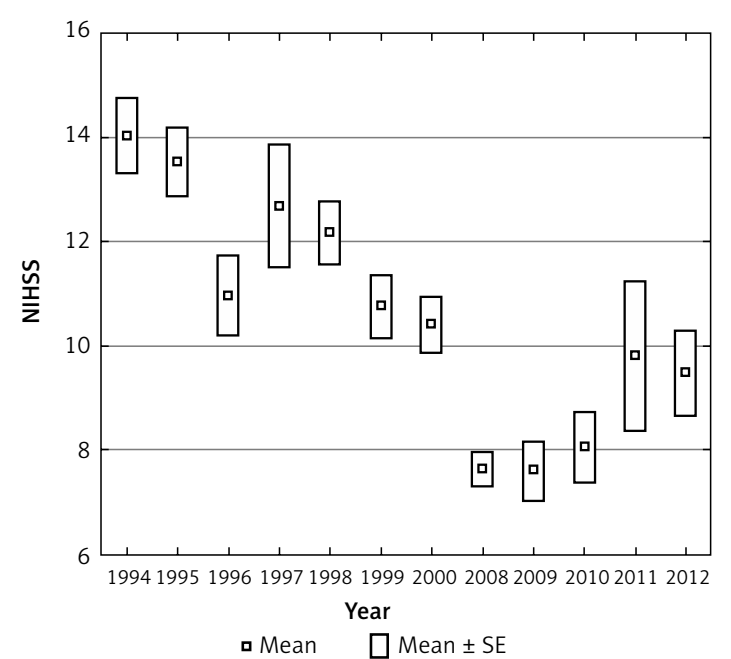

Figure 1. NIHSS scores in patients hospitalised in years $1994-2000$ and 2008-2012

\section{Results}

The characteristics of both cohorts are shown in Table I.

The patients hospitalised in the years 19942000 had a significantly higher NIHSS score on admission than those hospitalised in the years 2008-2012. The proportion of mild stroke was lower in the former. NIHSS scores are shown in Figure 1.

The distribution of risk factors and cardiovascular diseases was different between groups. The patients hospitalised in the years 2008-2012 more often had diagnosed hypertension, diabetes mellitus and atrial fibrillation than the patients hospitalised in the years 1994-2000. Those hospitalised in the years 1994-2000 more often suffered from hypercholesterolaemia and transient ischaemic attack (TIA) prior to stroke.

Both systolic and diastolic blood pressure values were higher on admission in the patients hospitalised in the years 1994-2000 than in those hospitalised in the years 1998-2012. The former more frequently had GFR below $60 \mathrm{ml} / \mathrm{min}$ per $1.73 \mathrm{~m}^{2}$ and more often had fasting hyperglycaemia.

Finally, 30-day case fatality was lower in the patients hospitalised in the years 2008-2012.

\section{Discussion}

We found decreased stroke severity reflected by a lower NIHSS score in the patients hospitalised in the years 2008-2012 compared with those hospitalised in the years 1994-2000. Stroke severity is the most important predictor of outcome. Thus, reduction in stroke severity could explain, at least partially, the lower case fatality observed by us in Polish stroke patients.

Paradoxically, the frequency of major risk factors (hypertension, diabetes mellitus, atrial fibrillation) was higher in the cohort hospitalised in the years 2008-2012. The exception was hypercholesterolaemia, which was less frequent in this cohort. Also the frequency of TIAs before stroke was lower in this group of patients. Higher frequency of major risk factors in the cohort hospitalised in the years 2008-2012 could be explained by better recognition and treatment of cardiovascular diseases in Poland in recent years [8]. We observed significantly lower values of both blood pressure on admission and fasting glucose in the patients hospitalised in the years 2008-2012. Although blood pressure and glucose level measured in acute stroke depend partially on stroke severity, their lower values also reflect better control of hypertension and hyperglycaemia before stroke. Furthermore, higher values of glomerular filtration rate (GRF), the recognised marker of subclinical organ damage in hypertension, in the patients hospitalised in the years 2008-2012 suggest better control of blood pressure in this group of patients.

Lower frequency of hypercholesterolaemia in patients hospitalised in the years 2008-2012 could be related to changes in dietary habits and/or more common use of statins. A reduction of total cholesterol was observed in the last decades in the Polish population and was attributed to increased consumption of oils rich in $\alpha$-linolenic acid [5-8].

Our study has several limitations. First, it was a single centre study, and our results do not necessarily reflect the epidemiological situation in the whole Polish population. Second, the conversion of SSS scores to NIHSS scores is not perfect, although a previous study showed satisfactory precision of this method [13]. Third, our study is not able to demonstrate causality between risk factors and stroke severity. Finally, we cannot verify the hypothesis that reduced stroke severity in our stroke patients may be related to more common use of statins and ACE inhibitors, drugs that could exert a neuroprotective effect [15-18]. Our database did not contain information about medications taken before stroke.

In conclusion, we observed a decrease of stroke severity among Polish patients hospitalised in recent years. The reduction in stroke severity was associated with better recognition and control of risk factors, which highlights the significance of primary prevention. However, it should be emphasised that the optimal blood pressure and cholesterol levels to optimise stroke prevention remain largely unknown $[19,20]$.

\section{Conflict of interest}

The authors declare no conflict of interest.

References

1. Ryglewicz D, Barañska-Gieruszczak M, Lechowicz W, et al. High case-fatality rates in the Warsaw stroke registry. J Stroke Cerebrovasc Dis 1997; 6: 421-5. 
2. Ryglewicz D, Hier DB, Wiszniewska M, et al. Ischemic strokes are more severe in Poland than in the United States. Neurology 2000; 54: 513-5.

3. Członkowska A, Niewada M, Saleh El-Baroni I, et al. High early case fatality after ischaemic stroke in Poland: exploration of possible explanations in the International Stroke Trial. J Neurol Sci 2002; 202: 53-7.

4. Czlonkowska A, Hier DB, Mendel T, et al. Heart disease contributes to high stroke mortality in Poland. J Stroke Cerebrovasc Dis 2000; 9: 76-8.

5. Zatonski WA, McMichael AJ, Powles JW. Ecological study of reasons for sharp decline in mortality from ischaemic heart disease in Poland since 1991. BMJ 1998; 316: 1047-51.

6. Zatonski WA, Willett W. Changes in dietary fat and declining coronary heart disease in Poland: population based study. BMJ 2005; 331: 187-8.

7. Zatonski W, Campos H, Willett W. Rapid declines in coronary heart disease mortality in Eastern Europe are associated with increased consumption of oils rich in alpha-linolenic acid. Eur J Epidemiol 2008; 23: 3-10.

8. Bandosz P, O'Flaherty M, Drygas W, et al. Decline in mortality from coronary heart disease in Poland after socioeconomic transformation: modelling study. BMJ 2012; 344: d8136.

9. Stowik A, Turaj W, Zwolińska G, et al. Stroke attack rates and case fatality in the Krakow Stroke Registry. Neurol Neurochir Pol 2007; 41: 291-5.

10. Sienkiewicz-Jarosz H, Głuszkiewicz M, Pniewski J, et al. Incidence and case fatality rates of first-ever stroke - comparison of data from two prospective population-based studies conducted in Warsaw. Neurol Neurochir Pol 2011; 45: 207-12.

11. Scandinavian Stroke Study Group. Multicenter trial of hemodilution in ischemic stroke. Stroke 1985; 16: 885-90.

12. Lyden P, Lu M, Jackson C, et al. Underlying structure of the National Institutes of Health Stroke Scale: results of a factor analysis. NINDS tPA Stroke Trial Investigators. Stroke 1999; 30: 2347-54.

13. Gray LJ, Ali M, Lyden PD, et al. Interconversion of the National Institutes of Health Stroke Scale and Scandinavian Stroke Scale in acute stroke. J Stroke Cerebrovasc Dis 2009; 18: 466-8.

14. Breuer L, Blinzler C, Huttner HB, et al. Off-label thrombolysis for acute ischemic stroke: rate, clinical outcome and safety are influenced by the definition of 'minor stroke. Cerebrovasc Dis 2011; 32: 177-85.

15. Greisenegger S, Müllner M, Tentschert S, et al. Effect of pretreatment with statins on the severity of acute ischemic cerebrovascular events. J Neurol Sci 2004; 221: 5-10.

16. Martínez-Sánchez P, Fuentes B, Martínez-Martínez M, et al. Treatment with statins and ischemic stroke severity: does the dose matter? Neurology 2013; 80: 1800-5.

17. Kumar S, Savitz S, Schlaug G, et al. Antiplatelets, ACE inhibitors, and statins combination reduces stroke severity and tissue at risk. Neurology 2006; 66: 1153-8.

18. Chitravas N, Dewey HM, Nicol MB, et al. Is prestroke use of angiotensin-converting enzyme inhibitors associated with better outcome? Neurology 2007; 68: 1687-93.

19. Malyszko J, Muntner P, Rysz J, Banach M. Blood pressure levels and stroke: J-curve phenomenon? Curr Hypertens Rep 2013; 15: 575-81.

20. Zanchetti A, Liu L, Mancia G, et al. G. Blood pressure and low-density lipoprotein-cholesterol lowering for prevention of strokes and cognitive decline: a review of available trial evidence. J Hypertens 2014; 32: 1741-50. 